https://doi.org/10.17816/MAJ191S127-28

\title{
CELLULAR MECHANISMS OF NEUROPSYCHIATRIC SYMPTOMS INDUCED BY HYPOTHYROIDISM IN MOUSE MODEL
}

\author{
Mami Noda \\ Laboratory of Pathophysiology, Graduate School of Pharmaceutical Sciences, Kyushu University
}

Thyroid hormones (THs) are essential not only for the development of the central nervous system (CNS) but also for matured brain function. In the CNS, circulating thyroxine (T4) crosses blood-brain barrier via specific transporters and is taken up to astrocytes, becomes L-tri-iodothyronine (3, 3', 5-triiodothyronine; T3), an active form of TH, by type 2 de-iodinase (D2). T3 is released to the brain parenchyma from astrocytes (glioendocrine system). In adult CNS, both hypo- and hyper-thyroidism, the prevalence in female being $>10$ times higher than that in male, may affect psychological condition and potentially increase the risk of cognitive impairment and neurodegeneration including Alzheimer's disease (AD). We have reported, that non-genomic effects of T3 on microglial functions and its signaling [1] and sex- and age-dependent effects of THs on glial morphology in the mouse brains of hyperthyroidism [2, 3]. Behavioral changes and spine density in hippocampus also showed sex-dependence. Recently we analyzed the opposite thyroid dysfunction, hypothyroidism, and found sex- and age-dependent changes in glial morphology and animal behavior as well. These results may help to understand physiological and/or pathophysiological functions of THs in the CNS and how hyper- and hypothyroidism affect psychological condition and cognition.

Keywords: hypothyroidism; thyroid hormones; Alzheimer's disease; glial cells.

\section{References}

1. Mori Y, et al. Effects of 3,3',5-triiodothyronine on microglial functions. Glia. 2015;63(5):906-920.

2. Noda M. Possible role of glial cells in the relationship between thyroid dysfunction and mental disorders. Front. Cell. Neurosci. 2015;9:194.

3. Noda M, et al. Sex- and age-dependent effects of thyroid hormone on glial morphology and function. $O M \& P .2016 ; 2: 85-92$. 